Flachot Alban

\title{
Extensions of a linear model of surface reflectance
}

Master's Thesis

July, 2016

Grenoble INP Phelma

Master IC2A 
Author: Flachot Alban

Contact information: flachot.alban@gmail.com

Supervisors: J. Kevin O’Regan, and Edoardo Provenzi

Title: Extensions of a linear model of surface reflectance

Project: Master's Thesis

Page count: $31+0$

\begin{abstract}
Philipona O'Regan recently proposed a linear model of surface reflectance as it is sensed by the human eyes. In their model, the three dimensional cone response to reflected light is accurately approximated by a linear transformation of the three dimensional response to illumination. The geometrical properties of this linear transformation, such as singularity, correlate with psychophysical results on focal colors and unique hues. Later, Vazquez-Corral et al. built a bridge between Philipona \& O'Regan's model and von Krieslike approaches to color constancy in computer vision by showing that the linear operators could be diagonalized in a common basis. However both of these studies required specifying a particular dataset of illuminants. We will show in this paper that it is possible to compute adequate linear operators and a common basis for diagonalization without specifying any particular set of illuminants, thus enhancing their generalizability to illuminant changes, while maintaining correlations with features of the human color vision. Further analysis of the characteristics of singularity in reflection properties will also be presented.
\end{abstract}

Keywords: Philipona and O'Regan, surface color, illuminant independance, linear operator, unique change of basis 


\section{List of Figures}

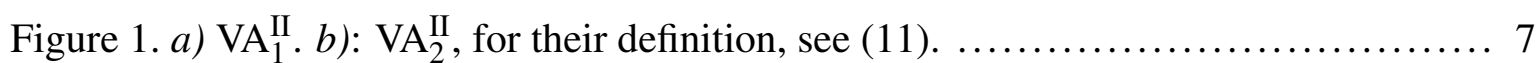

Figure 2. $a$ Reflectance curves giving a low VA 1 ..$b$ Reflectance curves giving a high

$\mathrm{VA}_{1}^{\mathrm{II}}$. Dashed curves are the Stockman \& Sharp cone fundamentals. $\ldots \ldots \ldots \ldots \ldots 8$

Figure 3. Case of the reflectance functions showing the lowest $\mathrm{VA}_{1}^{\mathrm{II}} . \ldots \ldots \ldots \ldots \ldots \ldots . \ldots$

Figure 4. Accuracy of the II appraoch with respect to Munsell hue, value and chroma..... 9

Figure 5. Plots of surface reflectances fo a given hue (RR) a) varying only in value

$($ chroma $=6)$ and $b)$ varying only in chroma (value $=4), \ldots \ldots \ldots \ldots \ldots \ldots \ldots \ldots 11$

Figure 6. Histogram of the relative commutativity for our set of matrices with mean

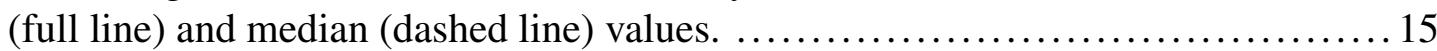

Figure 7. Gradient descent applied on the set of 2340 RMs computed using the II approach.17

Figure 8. Singularity index computed with PO's approach on the set of 320 munsell

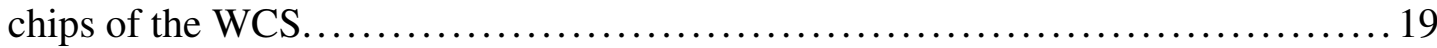

Figure 9. Singularity index of the 320 munsell chips computed with $a$ ) the II approach

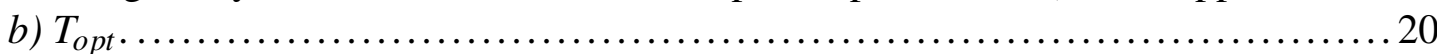

Figure 10. a) Singularity index according to hue, value and chroma for our set of 1600 Munsell chips. Small and dark bluish dots corresponds to small values of the singularity index while large and lighter yellowish dots to high values of the singularity index. $b$ ) Singularity index according to chroma and value. (1) 8 西 


\section{Contents}

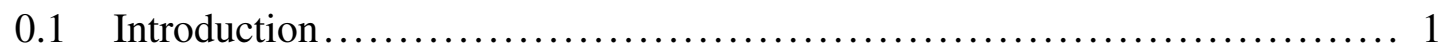

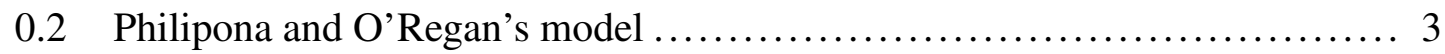

0.2 .1 Biological analogy of a surface reflectance....................... 3

0.2 .2 Diagonalisation of the reflectance matrix and computation of the vir-

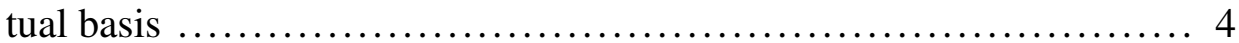

$0.3 \quad$ Analysis of the independence of Philipona \& O'Regan linear hypothesis

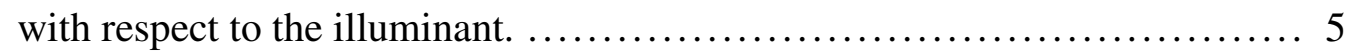

0.3.1 A novel approach to compute the RMs independently with respect

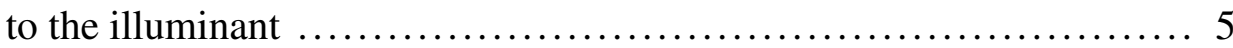

0.3 .2 Validity of the illuminant-independence of the linear hypothesis ........ 5

0.3 .3 Quantitative comparison between PO's and II approaches .............. 10

0.4 A theoretical and computational discussion of PO's model in the context

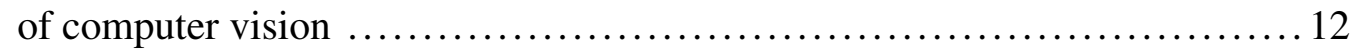

0.4 .1 Bridge between PO's model and computer vision ................... 12

0.4.2 Theoretical and empirical arguments for the existence of a unique virtual basis in the context of PO's framework...................... 14

0.4.3 A novel strategy to find a unique diagonalization matrix in PO's setting 16

0.5 Singularities in reflection properties and link with human color vision ........18

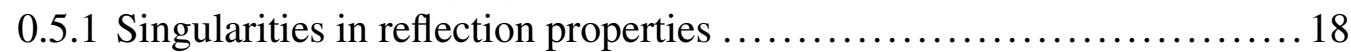

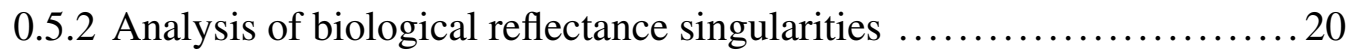

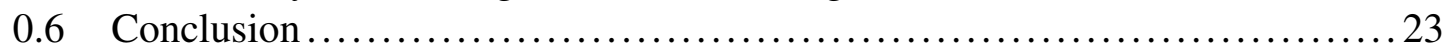

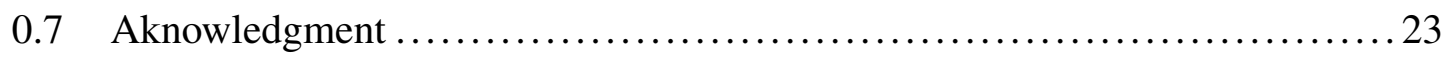

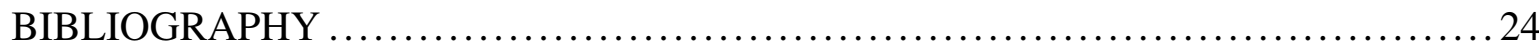

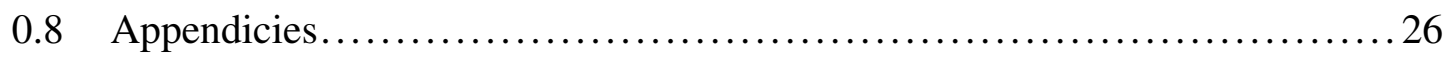

0.8 .1 Appendix A: Linear and Illuminant-Independant hypothesis . .......... 26

0.8 .2 Appendix B: Relative norm of a commutator ...................... 27 


\subsection{Introduction}

In physics, the spectrum of a natural light can be considered as composed of an infinite number of non-interactive monochromatic lights. In that sense, a light spectrum can be seen as a vector of infinite dimension, each dimension corresponding to a wavelength $\lambda$. Each component of the vector specifies the spectral power of the light at this wavelength $E(\lambda)$. In this framework, surfaces transform the incident light into the reflected light through a multiplicative coefficient $0 \leq S(\lambda) \leq 1$. This transformation is commonly called the surface reflectance function. However, daylight is only made available to the human nervous system through transformation of electromagnetic information into chemical information operated by the photopigments present in the three types of human cones, namely L, M and S (for long, medium and short wavelength). Such transfer of information can be described as a projection of a space of infinite dimension into a space of only three dimensions, each dimension corresponding to one type of cone (Koenderink and Doorn 2003). The available information (AI from now on) about light, available to the human nervous system at the retinal level, is thus a three dimensional vector. Since the $L$ and M cone sensitivity functions are highly overlapping, the $\mathrm{L}$ and $\mathrm{M}$ components of this space are highly interacting as well. (Philipona and O'Regan 2006) (PO from now on) studied the analogue of surface reflectance in this 3D space i.e. the transformation of the incident light into the reflected light as sensed by human eyes. They showed that this transformation can be well modeled by a linear operator, and hypothesized that this transformation is independent of the illuminant. They empirically confirmed this independence by statistically computing the linear operator using a set of natural illuminants. However, the fact that they specied a particular set of illuminants only guarantees partial independence. This limits the robustness of the model with respect to other illuminants.

In the first and second parts of this article, we will introduce PO's model in detail and present a novel computational/statistical approach to the model that is completely illuminantindependent. Quantitative comparison with PO will be discussed.

A third part of the article concerns a development of PO's approach by (Vazquez-Corral et al. 2012) (V-C from now on). These authors presented a development of PO in which they were able to empirically show that a global change of basis transform could be used to almost jointly diagonalize all the linear operators, represented by matrices, of PO's model. This transformation, through a linear combination, transforms human cone sensitivities into new ones which overlap less strongly, and are therefore less correlated. This procedure is very similar to spectral sharpening (Finlayson, Drew, and Funt 1994) and builds a bridge between PO's model and classic studies on color constancy.

Color constancy is a major feature of human color perception. It refers to the capacity of humans to naturally correct the effect of the illuminant on the light reflected by a surface. Since illuminant spectra, from natural to artificial, from morning to evening daylights, display a large range of shapes and amplitudes, the AI strongly varies with the illuminant change (Wyszecki and Stiles 1982). If human color perception solely depended on the AI, then the color appearance of an object would vary greatly with the illuminant as well. The fact that it 
does not, indicates that the human visual system succeeds, partially at least, in discounting the effect of the illuminant (Brainard, Kraft, and Longere 2003).

Color constancy has been extensively studied in human as well as in computer vision, and remains the subject of debate (Logvinenko et al. 2015). Many theories and approaches to color constancy attempt to estimate the illuminant from the visual scene (Land 1964; Van De Weijer and Gevers 2005; Provenzi et al. 2008), and then discount it from the AI. In this way they are able to extract intrinsic properties of the reflecting object (Wandel 1995), or to reconstruct the AI as if the object were illuminated by a fixed canonical light (Forsyth 1990). In computer vision, classic algorithms to achieve color constancy independently adjust the components of the AI (Von Kries 1902; Brill and West 1981; Land and McCann 1971). While this approximation is justified when the photoreceptor sensitivities can be considered disjoint, it fails when this is not so (Land 1983), as is the case for the L and M cones. Human $\mathrm{L}$ and $\mathrm{M}$ cone sensitivity functions do indeed overlap strongly. The $\mathrm{L}$ and $\mathrm{M}$ cone responses are thus highly correlated and cannot be considered independent. (Land 1983) suggested, in the general case of non-disjoint photoreceptor sensitivities, the use of a fixed linear combination of the photoreceptor functions in order to obtain new photoreceptor responses which are minimally correlated. (Finlayson, Drew, and Funt 1994) later applied this idea to human vision through their spectral sharpening approach. Psychophysical studies support the hypothesis that a linear combination of cone sensitivities is performed by the human visual system (for a review, see (Foster 1984)).

V-C's computation of a global sharpening transformation, however, also relies on the specification of a database of illuminants as well as an illuminant of reference. In the third part of this article, we will start by explaining thoroughly how the link between PO's model and classical strategies to achieve color constancy can be made through the definition of a global diagonalizing matrix. Then, we will use theoretical and computational arguments to justify the existence of such a surface and illuminant-independent transformation and finish by proposing a new method to compute this transformation.

A fourth part of the article concerns human color perception. Since color is naturally a characteristic of surfaces, geometrical properties of the linear operator modeling biological reflectances are expected to have an influence on human color perception. PO showed that the singularity of the matrices representing the linear operator may explain some features of color categorization, such as the particular perceptual status of so-called "focal" colors. Their computed singularity index correlates with the tendency of surface colors to be given names across diverse cultures (Berlin and Kay 1991). In this respect, PO's model contributes to the color naming and linguistic relativity debate, as to whether language influences color perception (the relativists' point of view) or whether color perception influences color naming (universalist point of view). For a review, see (Jraissati 2014). They were also able to correlate singularities of reflectances with unique hues i.e. hues that appear unmixed with others. In this fourth section of the article, we will recall the singularity index as defined in PO's paper as well as the correlation between singularities and psychophysical studies on focal colors and unique hues. We will then show that singularities of the matrices computed using our new illuminant-independent method, and additionally using a global change of ba- 
sis transformation, demonstrate the same correlations as PO. We will also detail the relation between singularities and some characteristics observed in the categorization of color such as lightness and saturation.

\subsection{Philipona and O'Regan's model}

PO studied the analogue of surface reflectances in the three dimensional space engendered by human sensors i.e. the transformation of the incident light into the reflected light as sensed by human eyes. They showed that this transformation can be well modeled by a linear operator, as described in the following section.

\subsubsection{Biological analogy of a surface reflectance}

Consider a surface $\mathbb{S}$ illuminated by a light with spectral power distribution $E(\lambda), \lambda \in \Lambda=$ $[400,700] \mathrm{nm}, \Lambda$ being the visual spectrum. Let $R_{L}(\lambda), R_{M}(\lambda), R_{S}(\lambda)$ denote the absorption rate at each wavelength $\lambda$ by photopigments present in the $L, M, S$ human photoreceptors. The information accessible to the human nervous system tahnks to the cones phototransduction can be expressed by $\mathbf{u}(E)=\left(u_{L}(E), u_{M}(E), u_{S}(E)\right)^{t}$, where:

$$
u_{i}(E)=\int_{\Lambda} R_{i}(\lambda) E(\lambda) d \lambda, \quad i=L, M, S .
$$

If we denote with $S(\lambda)$ the reflectance function of the surface $\mathbb{S}$, we can describe the accessible information about the light reflected by $\mathbb{S}$ as $\left.\mathbf{v}^{\mathbb{S}}(E)=\left(v_{L}^{\mathbb{S}}(E), v_{M}^{\mathbb{S}}(E), v_{S}^{\mathbb{S}}\right)(E)\right)^{t}$, with:

$$
v_{i}^{\mathbb{S}}(E)=\int_{\Lambda} R_{i}(\lambda) E(\lambda) S(\lambda) d \lambda, \quad i=L, M, S,
$$

we stress that this equation holds true only if there is no exchange of energy between different wavelengths (e.g. it does not hold for fluorescent surfaces). PO hypothesized that the transformation of $\mathbf{u}(E)$ into $\mathbf{v}^{\mathbb{S}}(E)$ is linear i.e.:

$$
\mathbf{v}^{\mathbb{S}}(E)=A^{\mathbb{S}} \mathbf{u}(E),
$$

where $A^{\mathbb{S}}: \mathbb{R}^{3} \mapsto \mathbb{R}^{3}$ is a linear operator that can be represented by a $3 \times 3$ matrix and which should only depend on the surface $\mathbb{S}$ and not on the illuminant $E$. We interpret $A^{\mathbb{S}}$ as the biological analogue of the physical reflectance of a surface, since it is the operator responsible for the transformation by a surface of the incident light into the reflected light, as sensed by the photoreceptors. We will thus call $A^{\mathbb{S}}$ the reflectance matrix (RM from now on).

The assumption of the existence of such a linear operator is not trivial. PO used statistical tools in order to verify it: they computed the empirical variance of the residuals $\eta=\mathbf{v}^{\mathbb{S}}(E)$ $A^{\mathbb{S}} \mathbf{u}(E)$, where $A^{\mathbb{S}}$ is obtained by linear regression of $\mathbf{v}^{\mathbb{S}}$ onto $u$ over a set of natural and simulated daylight illuminants (Philipona and O'Regan 2006). The percentage of variance accounted by the residuals for their set of surfaces (over 1600 Munsell chips and 1800 natural surfaces) are surprisingly low, with mean values lower than $0.5 \%$, which shows the validity of the the model and the linear hypothesis. 
We notice that, with this method, the matrix $A^{\mathbb{S}}$ depends on the dataset of illuminants used to compute it. In Section 0.3 we will discuss in greater detail this dependence and present a novel method for the computation of the RMs that allows complete independence with respect to the illuminant.

\subsubsection{Diagonalisation of the reflectance matrix and computation of the virtual basis}

PO showed that for every surface of their database, the RM $A^{\mathbb{S}}$ can either be exactly diagonalized or almost diagonalized with three distinct real eigenvalues. In other words, there exists an invertible matrix $T^{\mathbb{S}}$, which depends on the surface $\mathbb{S}$, such that:

$$
\mathbf{v}^{\mathbb{S}}(E)=\left(T^{\mathbb{S}}\right)^{-1} D^{\mathbb{S}} T^{\mathbb{S}} \mathbf{u}(E)
$$

where $D^{\mathbb{S}}$ is a diagonal $3 \times 3$ matrix. $T^{\mathbb{S}}$ is then the linear operator $: \mathbb{R}^{3} \rightarrow \mathbb{R}^{3}$ responsible for the change of basis where $A^{\mathbb{S}}$ is diagonal. This basis is given by the eigenvectors of $A^{\mathbb{S}}$.

Equation (4) can be rewritten as:

$$
\begin{aligned}
T^{\mathbb{S}} \mathbf{v}^{\mathbb{S}}(E) & =D^{\mathbb{S}} T^{\mathbb{S}} \mathbf{u}(E) \\
\tilde{\mathbf{v}}^{\mathbb{S}}(E) & =D^{\mathbb{S}} \tilde{\mathbf{u}}^{\mathbb{S}}(E),
\end{aligned}
$$

where $\tilde{\mathbf{u}}(E)$ and $\tilde{\mathbf{v}}^{\mathbb{S}}(E)$ are vectors in the new basis obtained by applying the operator $T^{\mathbb{S}}$. Note that, since $T^{\mathbb{S}}$ depends on the surface $\mathbb{S}$, so does $\tilde{\mathbf{u}}^{\mathbb{S}}(E)$.

By linearity and recalling Eq. $(1)$, we can write $T^{\mathbb{S}} u_{i}(E)=\int_{\Lambda} \sum_{j} T^{\mathbb{S}}{ }_{i j} R_{j}(\lambda) E(\lambda) d \lambda$, for every $i, j=L, M, S$. If we define $\widetilde{R}_{i}=\sum_{j} T^{\mathbb{S}}{ }_{i j} R_{j}(\lambda)$, we find:

$$
\tilde{u}_{i}^{\mathbb{S}}(E)=\int_{\Lambda} \tilde{R}_{i}(\lambda) E(\lambda) d \lambda, \quad i=L, M, S
$$

and likewise for $\tilde{v}_{i}^{\mathbb{S}}(E)$.

V-C (Vazquez-Corral et al. 2012) have interpreted $\tilde{R}_{i}$ as the absorption rate at each wavelength $\lambda$ of virtual photopigments present in virtual photoreceptors. For this reason, the basis in which the RM is diagonal is called the virtual basis, denoted by $\tilde{L} \tilde{M} \tilde{S}^{\mathbb{S}}$. The diagonality of the RM implies that the components of $\tilde{u}^{\mathbb{S}}(E)$ do not mutually interact with each other when they are reflected by the surface $\mathbb{S}$, and are simply individually scaled by the associated eigenvalues. In other words, the absorption rate curves of the virtual photopigments are less overlapping than the real ones, which is why they are called sharpened by finlayson1994spectral and V-C.

Then, by definition of the virtual basis we have $\tilde{v}_{i}^{\mathbb{S}}(E)=r_{i}^{\mathbb{S}} \tilde{u}_{i}^{\mathbb{S}}(E), i=\tilde{L}, \tilde{M}, \tilde{S}$, which is equivalent to:

$$
r_{i}^{\mathbb{S}}=\frac{\tilde{v}_{i}^{\mathbb{S}}(E)}{\tilde{u}_{i}^{\mathbb{S}}(E)} .
$$

$r_{\tilde{L}}^{\mathbb{S}}, r_{\tilde{M}}^{\mathbb{S}}, r_{\tilde{S}}^{\mathbb{S}}$ can thus be simply interpreted as three independent reflection coefficients in each of the three virtual channels. 


\subsection{Analysis of the independence of Philipona \& O'Regan linear hy- pothesis with respect to the illuminant.}

PO statistically computed the RM as the transformation of $\mathbf{u}(E)$ into $\mathbf{v}^{\mathbb{S}}(E)$. Doing so, their approach inherently relies on the specification of a database of illuminants, moreover natural in their case. As a consequence, the computed RM is only partially, instead of completely (cf Eq. (3)), illuminant-independent, and does not directly refer to the action of a surface on light defined in the three dimensional space given by human photoreceptors, whatever the illuminant may be. Computationally speaking, such a RM cannot accurately apply to an illuminant $E$ having little similarity with illuminants in the database used for the linear regression. In this Section, we will discuss a procedure to compute the matrices $A^{\mathbb{S}}$ without resorting to any particular database of illuminants. We will see that the technique we propose is a good trade-off between robustness with respect to illuminant changes and consistency of the matrices $A^{\mathbb{S}}$ with the linear model expressed by Eq. (3).

\subsubsection{A novel approach to compute the RMs independently with respect to the illumi- nant}

Let $A_{i j}^{\mathbb{S}}, j=L, M, S$, denote the components of $A^{\mathbb{S}}$. Then, if the linear hypothesis of PO holds true, we can easily explicitly write Eq. (3) in components as follows:

$$
v_{i}^{\mathbb{S}}(E)=\int_{\Lambda} E(\lambda) \sum_{j} A_{i j}^{\mathbb{S}} R_{j}(\lambda) d \lambda
$$

which, thanks to Eq. (2), can be rewritten :

$$
\int_{\Lambda} E(\lambda)\left(S(\lambda) R_{i}(\lambda)-\sum_{j} A_{i j}^{\mathbb{S}} R_{j}(\lambda)\right) d \lambda=0 .
$$

The other assumption of PO is that the RM should be independent of the illuminant, i.e. Eq. (9) must be true for any $E(\lambda)$. Thanks to the Fundamental Lemma of Calculus of Variations (Gelfand, Silverman, et al.2000), it follows that:

$$
S(\lambda) R_{i}(\lambda)-\sum_{j} A_{i j}^{\mathbb{S}} R_{j}(\lambda)=0, \quad \forall \lambda \in \Lambda .
$$

Thanks to Eq. (10), we can avoid performing a linear regression over a specified database of illuminants as in PO's method, because it can now be performed over $\Lambda$. We will call this approach the Illuminant Independent (II) approach.

\subsubsection{Validity of the illuminant-independence of the linear hypothesis}

In our calculations we used the same databases as PO did for natural illuminants (Romero, Garcia-Beltrán, and Hernández-Andrés 1997; Judd et al. 1964; Chiao, Cronin, and Osorio 2000) and for Munsell chips (Parkkinen, Hallikainen, and Jaaskelainen 1989), the latter made 
available by the University of Joensuu (http://spectral.joensuu.fi/). However, the database of natural surfaces that we used was taken from (Westland, Shaw, and Owens 2000), Joensuu and Trieste Universities. For cone sensitivities, we used the $2^{\circ}$ Stockman \& Sharp cone fundamentals (Stockman and Sharpe 2000) as in (Witzel, Cinotti, and O'Regan 2015) instead of the $10^{\circ}$ Stiles \& Burch (Stiles and Burch 1959) or the Smith \& Pokorny (Smith and Pokorny 1975) color matching functions used in PO's and V-C's papers respectively. The reason for our choice is simply that Stockman \& Sharp cone fundamentals directly refer to the sensitivity of human photoreceptors. In any case, the results are similar for all of the sensor functions chosen among those three.

Similarly to PO (Philipona and O'Regan 2006), we checked the validity of the linear and illuminant-independent hypothesis by computing the empirical variance of the residual $\eta=$ $y-\hat{y}$, where $y$ is the known quantity that the linear model tries to approximate and $\hat{y}$ is the approximated value obtained with the model. However, since we want the model to satisfy both Eq. (3) and Eq. (10), we considered the two kind of residuals defined in the following table:

\begin{tabular}{|c|c|c|}
\hline Residual & $y$ & $\hat{y}$ \\
\hline$\eta_{1}$ & $S(\lambda) \cdot \mathbf{R}(\lambda)$ & $A^{\mathbb{S}} \mathbf{R}(\lambda)$ \\
\hline$\eta_{2}$ & $\mathbf{v}^{\mathbb{S}}(E)$ & $A^{\mathbb{S}} \mathbf{u}(E)$, \\
\hline
\end{tabular}

where $\mathbf{R}(\lambda)=\left(R_{L}, R_{M}, R_{S}\right)(\lambda)$.

For further clarification, we used the exponents ${ }^{\mathrm{PO}}$ and ${ }^{\mathrm{II}}$ to differentiate the cases where the RMs have been computed with PO's or the II approach, respectively.

We will denote with $\mathrm{VA}_{i}^{k}$ the percentage of variance of data accounted for by the model, i.e.

$$
\mathrm{VA}_{i}^{k}=1-\operatorname{mean}_{L, M, S}\left(\frac{\operatorname{var}\left(\eta_{i}^{k}\right)}{\operatorname{var}\left(\mathbf{y}_{i}\right)}\right), \quad i=1,2, k=\mathrm{PO}, \mathrm{II} .
$$

Figure 1 and Table 1 summarize the results obtained with the II approach. In both graphs of Figure 1, the full and dashed lines represent the average and median value of $\mathrm{VA}_{i}^{k}$, respectively. The graph on the left refers to $\mathrm{VA}_{1}^{\mathrm{II}}$, while on the right we show that of $\mathrm{VA}_{2}^{\mathrm{II}}$.

The average values for the two graphs are $97 \%$ for $\mathrm{VA}_{1}^{\mathrm{II}}$ and $99.7 \%$ for $\mathrm{VA}_{1}^{\mathrm{II}}$. However, it is important to stress that the minimum attained by $\mathrm{VA}_{1}^{\mathrm{II}}$ is $50 \%$, while that of $\mathrm{VA}_{2}^{\mathrm{II}}$ is $92 \%$.

The results for $\mathrm{VA}_{1}^{\mathrm{II}}$ show that, even though the linear and illuminant-independent hypothesis hold true for the large majority of the reflectance functions considered, they do not for all of them. It is especially true for natural surfaces. However, the model still allows a very good approximation of the information about the reflected light $\mathbf{v}^{\mathbb{S}}(E)$, as shown by the results for VA $\mathrm{VA}_{2}^{\mathrm{II}}$. Examples of functions less accurately modeled by the II hypothesis are shown in Figure $2 \mathrm{a}$. For comparison, we plotted in Figure $2 \mathrm{~b}$ the 20 reflectance functions giving the best results. The dashed lines are the $2^{\circ}$ Stockman-Sharp cone fundamentals. One pattern 


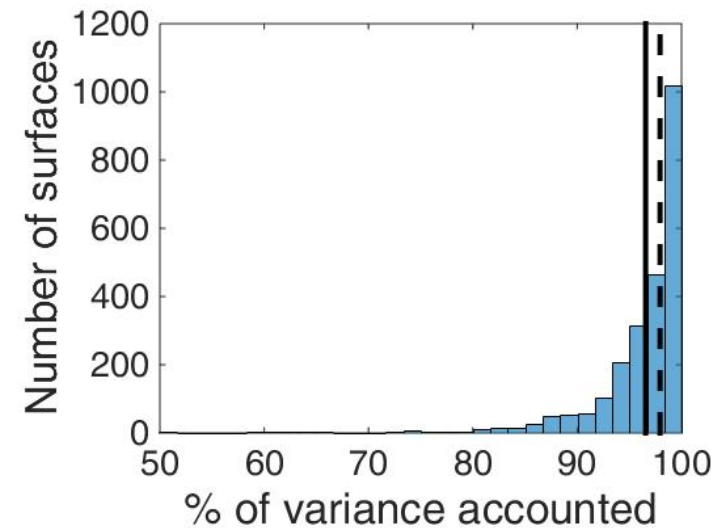

(a)

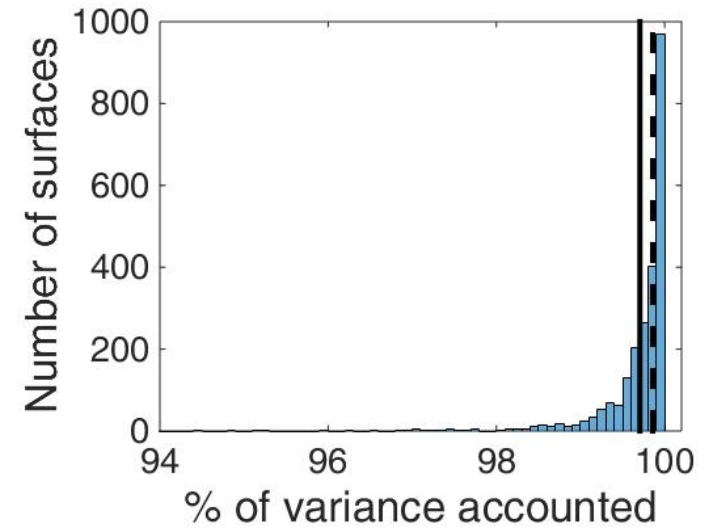

(b)

Figure 1. a) $\mathrm{VA}_{1}^{\mathrm{II}} . b$ ): $\mathrm{VA}_{2}^{\mathrm{II}}$, for their definition, see $(11$.

we can see when comparing these figures is the presence of high picks in the reflectance curves giving a low $\mathrm{VA}_{1}^{\mathrm{II}}$ while the reflectance curves giving a high $\mathrm{VA}_{1}^{\mathrm{II}}$ are almost constant over the visual spectrum (achromatic surfaces). And indeed, the ratio between the maximum of one reflectance curve and its minimum has a correlation coefficient of around .. with the errors in each case. In addition, it can be stressed from figure 2 that all of the reflectance curves leading to a low $\mathrm{VA}_{1}^{\mathrm{II}}$ have a peak either around the beginning and the end of the visual spectrum i.e. reflectance functions of red, purple or blue surfaces. These two parts of the spectrum correspond to intervals where only one of the cone fundamentals is particularly solicited by the reflected light, however not uniformly along its whole support due to the presence of a high peak in $S(\lambda)$. As a consequence, no linear combination of the two others cone sensitivities can be of influence and the linear model fails to give an accurate approximation.

For further illustration and a more detailed look at this limit of the linear hypothesis, Figure 3 shows an instance of these reflectance functions and its action on the S and L cone sensitivities. The dotted line corresponds to the reflectance curve, the full lines correspond to the two products $S(\lambda) R_{i}(\lambda), i=S, L$ while the dashed lines are the results given by the linear regression as the most suited linear combinations of the cone response to fit the full lines. It appears clearly, especially in the case of the L-cone, how the model fails to give a good approximation. However, the figure also illustrates why, even in this case, VA ${ }_{2}^{\text {II }}$ can still be satisfactory: due to a property of the linear regression, the surfaces under the dashed and full lines are equivalent. Therefore, so are their integrals. Natural illuminant spectra being broad band and rather smooth on $\Lambda$ (Romero, Garcia-Beltrán, and Hernández-Andrés 1997), the difference between the two terms of Eq. (9) compensate each other under the integral and give similar values. This reasoning holds obviously only in the case of smooth illuminant spectra such as the natural ones, and not if we consider monochromatic illuminants for example. This is illustrated by the cyan vertical line in Figure 3. While the L-component of $\mathbf{v}^{\mathbb{S}}(E)$ is obviously non zero (product of the vertical line with the full blue curve), the model gives a null value (product of the vertical line with the dashed blue curve) and thus fails to 


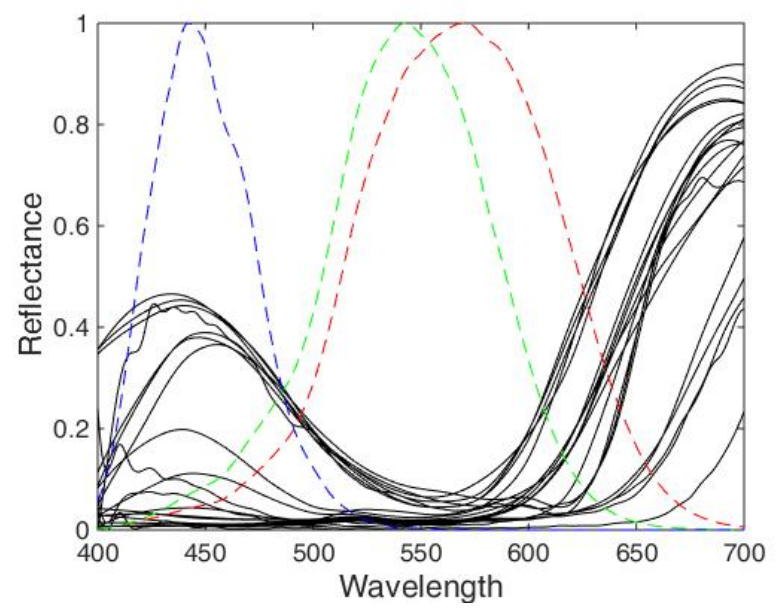

(a)

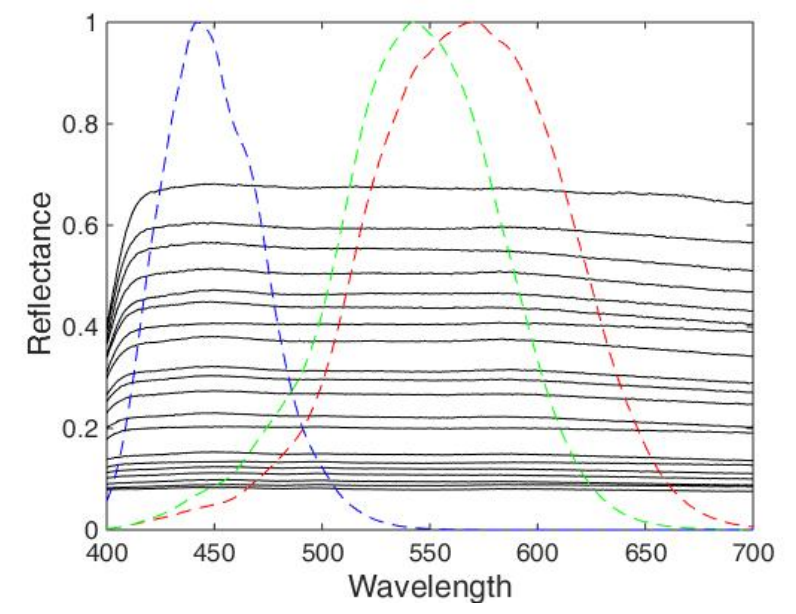

(b)

Figure 2. $a$ Reflectance curves giving a low VA $\mathrm{VA}_{1}^{\mathrm{II}}$. $b$ Reflectance curves giving a high VA $\mathrm{II}_{1}$. Dashed curves are the Stockman \& Sharp cone fundamentals.

give a correct approximation.

In order to correlate some general properties of surface reflectances with their perceptual characteristics and the limits of the linear and illuminant-independent hypothesis, we considered the set of 1600 Munsell chips organized following the Munsell color system. This system, first proposed by munsell1912pigment, is a standard color space widely used in colorimetry and for psychophysical studies representing color with cylindrical coordinates, in three independent and perceptually uniform dimensions: hue, value and chroma. The value, or lightness, is the vertical axis, ranging from 0 (black) to 10 (white), and is directly related to the amount of light transmitted by the colored surface. Originally, and as a first approximation, the value is proportional to the square toot of the luminance (Malacara 2011). The hue of the color is the polar angle and is divided into 10 main categories: red (RR or R), yellow-red (YR) yellow (YY or Y), green-yellow (GY), yellow-red (YR), green (GG or G), 


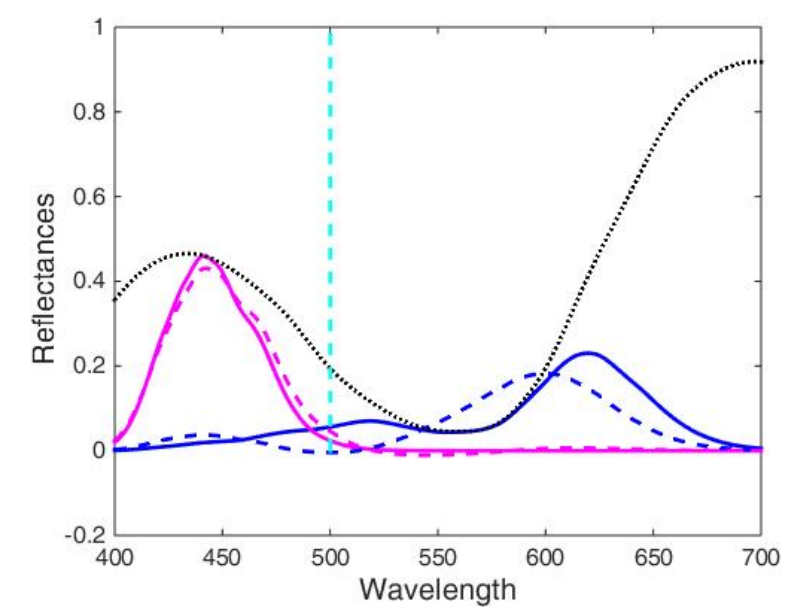

Figure 3. Case of the reflectance functions showing the lowest $\mathrm{VA}_{1}^{\mathrm{II}}$.

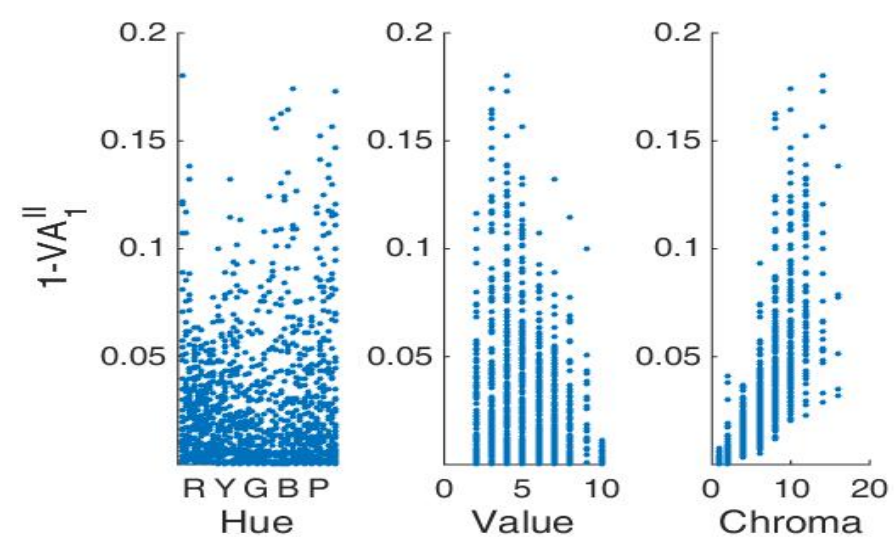

Figure 4. Accuracy of the II appraoch with respect to Munsell hue, value and chroma.

blue-green (BG), blue (BB or B), purple-blue (PB), purple (PP or P) and red-purple (RP). Each of the main categories are divided into 4 subcategories: 2.5, 5, 7.5 and 10. Chroma, or color strength, or saturation, is given by the distance to the value axis. It is divided into 8 values, from 2 to 16 by steps of 2 . Chroma accounts for the "purity" of a hue, purity being its amount of visual difference from a grey of the same value. The lower the chroma the less pure the color. A particularity of the Munsell color system worth pointing out is its irregular volume: neither spherical nor rectangular, the highest saturations that can be obtained depend on the value and the hue of the particular chips.

Figure 4 displays the dependence of the linear and illuminant-independant hypothesis on hue, value and chroma. The vertical axis represents the variance accounted for by the residuals $1-\mathrm{VA}_{1}^{\mathrm{II}}$ (see Eq. (11)). We can see on the left plot that, even though at first sight the performance of the linear regression is poorly correlated with the hue of the surface, it still confirms the previous observation: that the approximation is less accurate for some red, blue and purple surfaces. The center plot shows that value may have some influence as well. The 


\begin{tabular}{|c|c|c|c|c|c|c|c|c|c|}
\hline & \multicolumn{3}{|c|}{$\mathrm{VA}_{1}^{\mathrm{II}}$} & \multicolumn{3}{c|}{$\mathrm{VA}_{2}^{\mathrm{II}}$} & \multicolumn{3}{c|}{$\mathrm{VA}_{2}^{\mathrm{PO}}$} \\
\hline & mean & median & min & mean & median & min & mean & median & min \\
\hline natural & 96.57 & 97.95 & $\mathbf{5 0 . 2 5}$ & 99.70 & 99.96 & $\mathbf{9 1 . 9 3}$ & 99.94 & 99.97 & $\mathbf{9 8 . 8 3}$ \\
\hline fluorescent & $"$ & $"$ & $"$ & 99.45 & 99.75 & $\mathbf{8 7 . 2 3}$ & 98.69 & 99.45 & $\mathbf{6 5 . 5 3}$ \\
\hline monochromatic & $"$ & $"$ & $"$ & 96.33 & 97.83 & $\mathbf{3 7 . 7 2}$ & 91.68 & 96.00 & $\mathbf{- 2 . 3 0}$ \\
\hline
\end{tabular}

Table 1. VA values in the case of natural, florescent and almost-monochromatic illuminants. See text for further explanations.

most striking dependence however is with chroma, with a correlation of 0.78 .

The value of a Munsell chip is by definition only dependent on the amount of light transmitted by the surface. In other words, two colored surfaces of the Munsell system, different only in Munsell value by a factor $k$, will have at first approximation the same aspect, homogeneously scaled by a scalar multiplicative factor $C(k)$ that depends only on k (Cf figure $5 \mathrm{a}$ ). Thus, if we denote $S_{h, v, c}(\lambda)$ the reflectance function of a Munsell chip of hue $h$, value $v$ and chroma $c$, we can write:

$$
S_{h, k v, c}(\lambda)=C(k) S_{h, v, c}(\lambda) .
$$

Since the multilinear regression of Eq. (10) fits the product of the reflectance function with the cone sensitivities via a linear combination of the three cone sensitivity functions, any set of reflectances, different only by a multiplicative constant will therefore allow the same fitting accuracy. This is the reason why the munsell value is of poor influence in the accuracy of the linear and independent approximation.

Chroma, on the other hand, deforms in-homogeneously the reflectance functions as seen in figure $5 \mathrm{~b}$ ) by enhancing the contrast in reflectance between wavelength. The higher the chroma, the further we get from an achromatic surface reflectance curve. Thus, chroma inhomogeneously deforms the $S(\lambda) \mathbf{R}(\lambda)$ term of Eq.(3) and makes it more difficult to linearly approximate.

Thus, the relation between the incident and the reflected light as sensed by humans cannot be considered both linear and illuminant-independent for every surface, especially for high saturated red, blue and purple surfaces. Nonetheless, even for these surfaces, the computed RM allows a fairly accurate approximation of $\mathbf{v}^{\mathbb{S}}(E)$ when $E$ is a natural illuminant, as confirmed by the values of $\mathrm{VA}_{2}^{\mathrm{II}}$.

\subsubsection{Quantitative comparison between PO's and II approaches}

Looking at Table 1 and comparing the $\mathrm{VA}_{2}^{\mathrm{II}}$ and $\mathrm{VA}_{2}^{\mathrm{PO}}$ obtained for natural illuminants, one could argue that the $A^{\mathbb{S}}$ computed with PO's approach allows far better results than with the II approach. But the reason for this is that since the regression matrices $A^{\mathbb{S}}$ calculated with PO's strategy are computed with respect to a particular set of illuminants, natural in this case, they are optimally adapted to it, while those computed with the II approach are not. Figure shows the action of a reflectance function, similar to the one discussed in the previous 


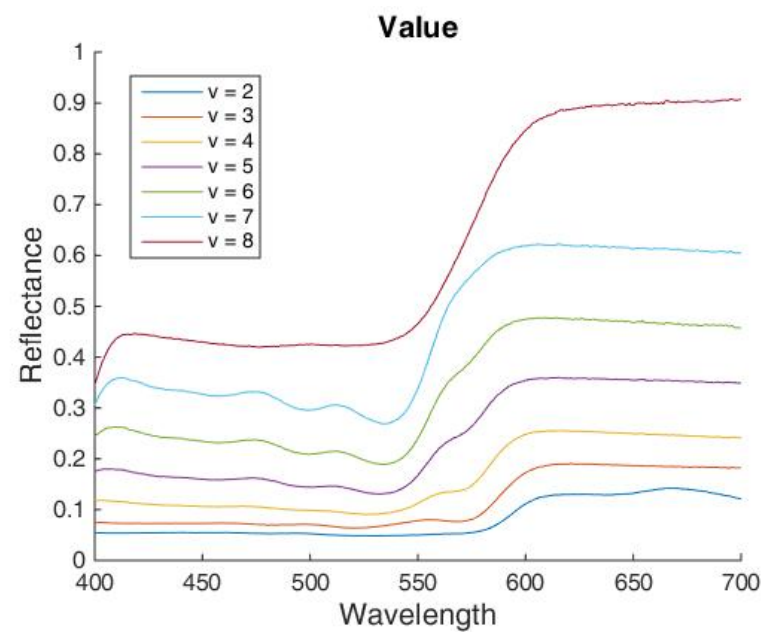

(a)

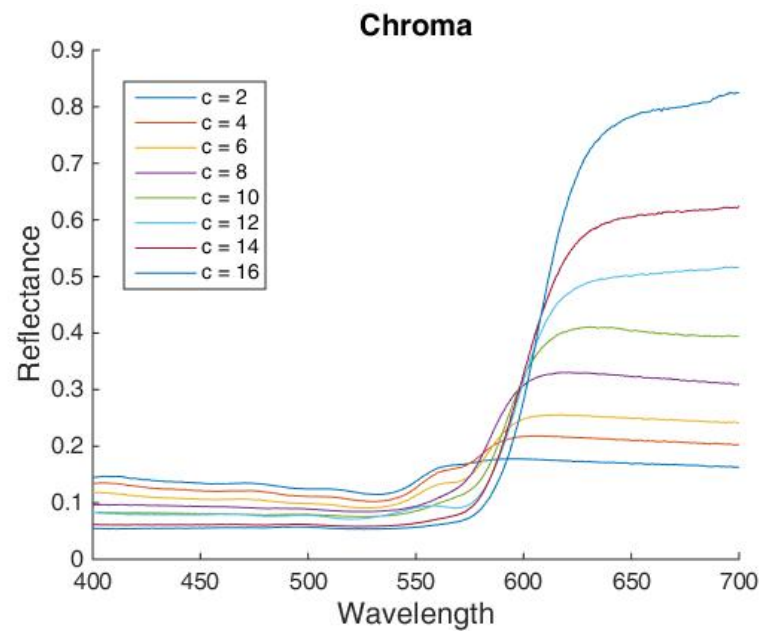

(b)

Figure 5. Plots of surface reflectances fo a given hue (RR) a) varying only in value (chroma $=6)$ and $b$ ) varying only in chroma (value $=4$ ).

section, on the $\mathrm{L}$ and $\mathrm{S}$ cone sensitivities, as well as the approximation given by the model using PO's approach to compute the corresponding RM.

Thus, we thought it would be interesting to test the robustness of the two approaches with respect to illuminant change i.e the consistency between the $A^{\mathbb{S}}$ matrices and the linear model in Eq. (3) when a drastic change of E occurs. In order to test this, we considered two other illuminant datasets provided by Witzel et al in (Witzel, Cinotti, and O'Regan 2015). The first one was of fluorescent illuminants and the second was of almost-monochromatic visual lights. Both types of illuminants are very different from the natural ones.

In Table 1, we show the VA values with the II and PO approaches for natural, florescent and almost-monochromatic illuminants. The key point is that all values of $\mathrm{VA}_{2}^{\mathrm{PO}}$ were calculated with the RMs computed over the dataset of natural illuminants and applied to the fluorescent 
and almost-monochromatic conditions.

We notice that PO's matrices become less and less adapted to satisfy the linear model when we pass from the natural illuminant dataset to the florescent and the almost-monochromatic ones, with $\mathrm{VA}_{2}^{\mathrm{PO}}$ average values of 99,9 to 98.7 to 91.7 respectively. In particular, we observe the presence of a negative value for $\mathrm{VA}_{2}^{\mathrm{PO}}$. Instead, the $A^{\mathbb{S}}$ matrices computed with the II model remain, in terms of mean, median and minimal value, a better approximation than PO's for the florescent and almost-monochromatic sets. Once computed, the RMs found with the II approach are thus more reliable than those found with PO's in terms of generalisability to other illuminants. In this way, they more directly refer to the action of a surface in the three dimensional space given by the human photoreceptors, independently of the illuminant.

\subsection{A theoretical and computational discussion of PO's model in the context of computer vision}

In their article, PO pointed out the possible link of the model with spectral sharpening (Finlayson, Drew, and Funt 1994). They argued that this link is valid if the matrices $A^{\mathbb{S}}$ can be diagonalized using a unique transformation that is independent of the surface $\mathbb{S}$. V-C later computed a global transformation which role is very similar to jointly diagonalizing all matrices, however still relying on the same database of illuminant as PO, and in addition relying on a reference illuminant, the CIE standard illuminant $D_{65}$ in the present case. They also limited their database of surfaces to the 320 colored munsell chips used in the WCS studies (Berlin and Kay 1991). For the sake of clarity, we will start this section by describing thoroughly the bridge between PO's model and computer vision. Then, we will show that the existence of a $\mathbb{S}$-independent diagonalizing transformation is a theoretical consequence of PO's model applied to multiple surfaces. Finally, using the large set of RM computed thanks to the II approach, we will present a novel strategy to compute this transformation without resorting to any standard illuminant or any particular illuminant database thanks to a gradient descent.

\subsubsection{Bridge between PO's model and computer vision}

Suppose that we have a visual scene illuminated by only one spatially homogeneous light source $E(\lambda)$, and consider an RGB camera with spectral sensitivity functions picked in the low, medium and high visible wavelengths, usually indicated with $R, G$ and $B$, respectively. We denote the spectral sensitivity functions as $\rho_{c}: \Lambda \rightarrow \mathbb{R}^{+}, c \in\{R, G, B\}$. The analysis performed in (Jiang et al. 2013) showed that $\operatorname{supp}\left(\rho_{c}\right)$, the supports of the functions $\rho_{c}$, are very similar and weakly overlapping compared to the spectral sensitivity functions of retinal cones.

In the Lambertian digital image formation model, see e.g. (Gijsenij, Gevers, and Van De Weijer 2011), the intensity $v_{c}(x, E)$ of a pixel $x$ in the chromatic channel $c$ is represented as 
follows:

$$
v_{c}(x, E)=\int_{\Lambda} \rho_{c}(x, \lambda) S(x, \lambda) E(\lambda) d \lambda .
$$

If we consider the supports $\operatorname{supp}\left(\rho_{c}\right)$ as not overlapping and the reflectance functions $S(x, \lambda)$ as constant with respect to $\lambda$ in each subset $\operatorname{supp}\left(\rho_{c}\right)$, i.e. $S(x, \lambda) \equiv S_{c}(x)$ for all $\lambda \in \operatorname{supp}\left(\rho_{c}\right)$ and $c \in\{R, G, B\}$, then $v_{c}(x, E)$ can be re-written as:

$$
\left(\begin{array}{l}
v_{R}(x, E) \\
v_{G}(x, E) \\
v_{B}(x, E)
\end{array}\right)=\left(\begin{array}{ccc}
S_{R}(x) & 0 & 0 \\
0 & S_{G}(x) & 0 \\
0 & 0 & S_{B}(x)
\end{array}\right)\left(\begin{array}{l}
u_{R}(E) \\
u_{G}(E) \\
u_{B}(E)
\end{array}\right) .
$$

Notice that the simultaneous dependence on $x$ and $E$ of the left-hand side of the equation is separated into two multiplicative components, one being the diagonal matrix of elements $S_{c}$ which depends on $x$, and the other being the vector components $u_{c}$ which depend on $E$.

The von Kries diagonal transformation (Von Kries 1902), used as a basis in several approaches to color constancy, see e.g. (West and Brill 1982; Forsyth 1990), is described by the following formula:

$$
\left(\begin{array}{l}
S_{R}(x) \\
S_{G}(x) \\
S_{B}(x)
\end{array}\right)=\left(\begin{array}{ccc}
1 / u_{R}(E) & 0 & 0 \\
0 & 1 / u_{G}(E) & 0 \\
0 & 0 & 1 / u_{B}(E)
\end{array}\right)\left(\begin{array}{l}
v_{R}(x) \\
v_{G}(x) \\
v_{B}(x)
\end{array}\right) .
$$

We stress that the von Kries matrix transformation is diagonal thanks to the diagonal nature of Eq. (14). Moreover, its independence with respect to the pixel position $x$ is a consequence of the separability property of Eq. (14).

We would like to translate what we have just stated into the terms of PO's model. First of all, the equivalent of Eq. (14) in PO's setting is Eq. (3). The matrices appearing in the two formulae depend only on surface properties. However $A^{\mathbb{S}}$ is not diagonal, thus it is not possible to write a von Kries-like transformation to obtain information on the reflectance.

Notice however, that in the virtual basis expressed by Eq. (5) the RM is diagonal. If we write Eq. (5) in terms of components we find:

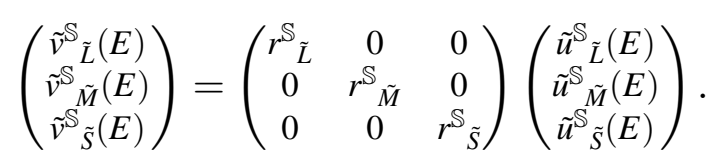

By direct computation, the previous formula can be re-written as:

$$
\left(\begin{array}{c}
r_{\tilde{L}}^{\mathbb{S}_{\tilde{L}}} \\
r_{\tilde{\mathbb{S}}}^{\mathbb{S}_{\tilde{M}}} \\
r_{\tilde{S}}^{\mathbb{S}}
\end{array}\right)=\left(\begin{array}{ccc}
1 / \tilde{u}_{\tilde{L}}^{\mathbb{S}}(E) & 0 & 0 \\
0 & 1 / \tilde{u}_{\tilde{M}}^{\mathbb{S}}(E) & 0 \\
0 & 0 & 1 / \tilde{u}_{\tilde{S}}^{\mathbb{S}}(E)
\end{array}\right)\left(\begin{array}{c}
v_{\tilde{L}}^{\mathbb{S}}(E) \\
v_{\tilde{S}}^{\mathbb{S}_{\tilde{M}}(E)} \\
v_{\tilde{S}}^{\mathbb{S}(E)}
\end{array}\right) .
$$

If we look at Eqs. (15) and (17) we see that in the latter the diagonal matrix depends on the surface $\mathbb{S}$, as a consequence of the dependence of $\tilde{\mathbf{u}}^{\mathbb{S}}=T^{\mathbb{S}} \mathbf{u}$ on $\mathbb{S}$. This argument shows that, if we want to be able to apply a von Kries-like transformation in PO's framework, the transformation $T^{\mathbb{S}}$ to the virtual basis must be independent of $\mathbb{S}$.

In the following subsections we will discuss this important issue in theoretical and computational terms. 


\subsubsection{Theoretical and empirical arguments for the existence of a unique virtual basis in the context of PO's framework}

The existence of a global change of basis transform can be theoretically derived from Eq. (1), (2) and (3), the very roots of PO's model. First of all, let us recall an important result of linear algebra: a set of matrices is simultaneously diagonalizable if and only if they commute. In particular, in PO's setting, this means that two matrices $A^{\mathbb{S}_{1}}$ and $A^{\mathbb{S}_{2}}$, relative to two arbitrary surfaces $\mathbb{S}_{1}$ and $\mathbb{S}_{2}$, can be diagonalized in the same basis if and only if they commute, i.e. $A^{\mathbb{S}_{1}} A^{\mathbb{S}_{2}}=A^{\mathbb{S}_{2}} A^{\mathbb{S}_{1}}$. We shall now give an argument to show that the commutativity of these matrices must indeed hold true. First of all, notice that, from a physical point of view, light reflected by a surface of spectral reflectance $S(\lambda)$ is indistinguishable from light emitted by a source whose power distribution is $E(\lambda) S(\lambda)$. Considering Eqs. (1) and (2), this can be translated into the following expression:

$$
\begin{aligned}
v_{i}^{\mathbb{S}}(E) & =\int_{\Lambda} R_{i}(\lambda)[S(\lambda) E(\lambda)] d \lambda, \quad i=L, M, S \\
& =u_{i}(S \cdot E) .
\end{aligned}
$$

Thus, if we consider the situation of a ray of light reflected by a surface $\mathbb{S}_{1}$ onto another surface $\mathbb{S}_{2}$, then due to the definition of reflectance the accessible information about the light reflected in this process is:

$$
\begin{aligned}
u_{i}\left(S_{1} S_{2} \cdot E\right) & =\int_{\Lambda} R_{i}(\lambda)\left[S_{1}(\lambda) S_{2}(\lambda) E(\lambda)\right] d \lambda \\
& =\int_{\Lambda} R_{i}(\lambda)\left[S_{2}(\lambda) S_{1}(\lambda) E(\lambda)\right] d \lambda \\
& =u_{i}\left(S_{2} S_{1} \cdot E\right),
\end{aligned}
$$

which shows that the role of $\mathbb{S}_{1}$ and $\mathbb{S}_{2}$ can be inverted in the process described above.

Notice now that we can write in its vector form:

$$
\begin{aligned}
\mathbf{u}\left(S_{2} S_{1} \cdot E\right) & =\mathbf{v}^{\mathbb{S}_{2}}\left(S_{1} \cdot E\right)=A^{\mathbb{S}_{2}} \int_{\Lambda} \mathbf{R}(\lambda) S_{1}(\lambda) E(\lambda) d \lambda \\
& =A^{\mathbb{S}_{2}} A^{\mathbb{S}_{1}} \mathbf{u}(E) .
\end{aligned}
$$

Similarly, if we compute $\mathbf{u}\left(S_{1} S_{2} \cdot E\right)$ we obtain $A^{\mathbb{S}_{1}} A^{\mathbb{S}_{2}} u(E)$. Thanks to Eq. [19, we have $A^{\mathbb{S}_{1}} A^{\mathbb{S}_{2}} u(E)=A^{\mathbb{S}_{2}} A^{\mathbb{S}_{1}} u(E)$ for all pairs of surfaces $\mathbb{S}_{1}$ and $\mathbb{S}_{2}$, hence the commutativity of the RM matrices and the existence of a global change of basis transform. Note that this is only true theoretically if and only if Eq. (3) holds exactly. However, as seen in the previous section, it is not the case. We thus calculated the commutativity of our set of 2340 matrices to test whether with the matrices computed with the II approach, the existence of a global change of basis is to be expected. As showed in appendix 0.8.2, if we define the commutator $C_{i j}$ as:

$$
C_{i j}=A^{\mathbb{S}_{i}} A^{\mathbb{S}_{j}}-A^{\mathbb{S}_{j}} A^{\mathbb{S}_{i}}
$$

we can define a criterion $R_{i j}$ for the relative commutativity:

$$
R_{i j}=\frac{\left\|C_{i j}\right\|}{2\left\|A_{i}^{\mathbb{S}}\right\|\left\|A_{j}^{\mathbb{S}}\right\|} \leq 1, \quad \forall i, j \in\{1,2, \ldots, 2340\}
$$




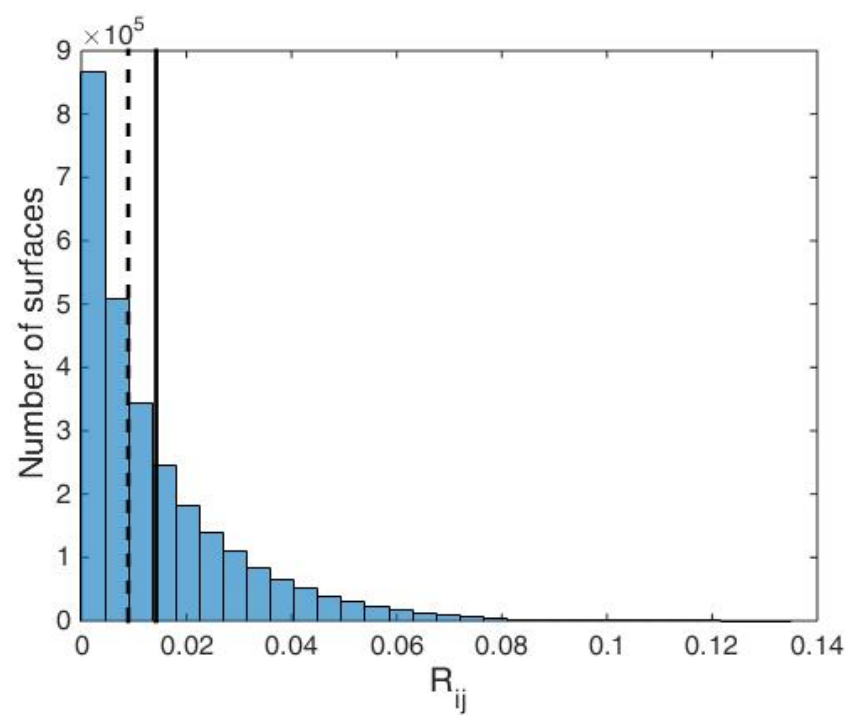

Figure 6. Histogram of the relative commutativity for our set of matrices with mean (full line) and median (dashed line) values.

We thus plotted the left part of equation 22 in figure 6 . We found that in most cases the commutators are indeed very small, with a mean and median of around 0.014 and 0.01 respectively. It means indeed that for the major part of the matrices, choosing a global change of basis transformation would expectedly do little difference in the diagonal coefficients of the resulting matrix than if an optimal change of basis transformation was used for each. Even though this statement has not yet been mathematically proven for real valued nonsymmetric matrices, as the RM, it has been proven for other cases (O'Meara and Vinsonhaler 2006) and empirically verified (Bunse-Gerstner, Byers, and Mehrmann 1993) (Cardoso and Souloumiac 1996). However figure 6 shows that for some commutators $R_{i j}$ is significantly high, with a value higher than 0.1 i.e. to more than $10 \%$ of its superior limit. A threshold needs yet to be defined in order to characterize whether a commutator is significant or not.

We argued that if Eq. (3) holds perfectly true, then all RM perfectly commute, therefore the lack of commutativity should be a consequence of the limits of the linear and II hypothesis of the RM; In order to verify this, we calculated the correlation coefficients between the variance accounted by the residuals $1-\mathrm{VA}_{1}^{\mathrm{II}}$ (cf section 0.3.2) and the normalized mean commutator's norm obtained for each RM. We found a coefficient of correlation of around 0.7 , which clearly assesses the relation between the lack of commutativity and the lack of accuracy of the linear model.

In any case, the commutativity of our set of matrices, even though very large, allow the statement of the existence of a global change of basis matrix that nearly diagonalize all RM. 


\subsubsection{A novel strategy to find a unique diagonalization matrix in PO's setting}

Now that we have provided an argument for the existence of a unique transformation $T$ able to jointly almost diagonalize all RM matrices at once, we have to computationally find it.

This problem has already been considered by V-C et al. in (Vazquez-Corral et al. 2012), where the authors proposed a strategy to find $T$ which, however, once again depends on the choice of a particular illuminant and on the database that was selected.

Here we are going to describe a new strategy that does not have these limitations.

A commonly used measure for the the joint diagonality of a set of $N$ matrices $\left\{M^{k}\right\}_{k=1}^{N}$ proposed in (Bunse-Gerstner, Byers, and Mehrmann 1993) is the average of the Frobenius norm of the off-diagonal elements of each matrix, i.e.

$$
\begin{aligned}
J D & =\frac{1}{N} \sum_{k=1}^{N}\left(\sum_{i \neq j}\left|M_{i j}^{k}\right|^{2}\right)^{1 / 2} \\
& =\frac{1}{N} \sum_{k=1}^{N}\left\|\left[M^{k}-\operatorname{diag}\left(M^{k}\right)\right]_{i j}\right\|_{\mathrm{FRO}} .
\end{aligned}
$$

The larger $J D$, the less the set of matrices is jointly diagonal.

The goal of the algorithm that we want to propose is thus to find the optimal change of basis transformation $T_{\mathrm{opt}}$ that minimizes $J D$. Using the notations introduced for PO's framework in the case of $N$ surfaces $\mathbb{S}_{k}$, we can write the optimality condition as follows:

$$
\begin{aligned}
T_{\mathrm{opt}} & =\underset{T \in \mathbb{R}^{3 \times 3}}{\arg \min } J D(T) \\
& =\underset{T \in \mathbb{R}^{3 \times 3}}{\arg \min } \frac{1}{N} \sum_{k=1}^{N} \sum_{i \neq j}\left|\left(T^{-1} A^{\mathbb{S}_{k}} T\right)_{i j}\right|^{2} .
\end{aligned}
$$

We stress that the matrices that appear in the previous equation are not symmetric nor selfadjoint, thus we cannot apply the minimization strategies proposed in (Bunse-Gerstner, Byers, and Mehrmann 1993; Cardoso and Souloumiac 1996; Afsari and Krishnaprasad 2004; Ziehe et al. 2004; Tichavsk and Yeredor 2009) in our context. However, the gradient descent strategy proposed by (Hori, 1999) (Hori 1999) can be adapted to our framework because it requires only the non-singularity of matrices.

The gradient of $J D$ is:

$$
\nabla J D(T)=2 T \sum_{k=1}^{N}\left[\left(T^{-1} A^{\mathbb{S}_{k}} T\right)^{t},\left(T^{-1} A^{\mathbb{S}_{k}} T-\operatorname{diag}\left(T^{-1} A^{\mathbb{S}_{k}} T\right)\right)\right],
$$

where $\left[M_{1}, M_{2}\right]=M_{1} M_{2}-M_{2} M_{1}$ is the commutator between matrices, so the discrete gradient descent equation to find $T_{\mathrm{opt}}$ is:

$$
T_{n+1}=T_{n}-\alpha_{n} \frac{\nabla J D\left(T_{n}\right)}{\left\|\nabla J D\left(T_{n}\right)\right\|}, \quad n \geq 0
$$




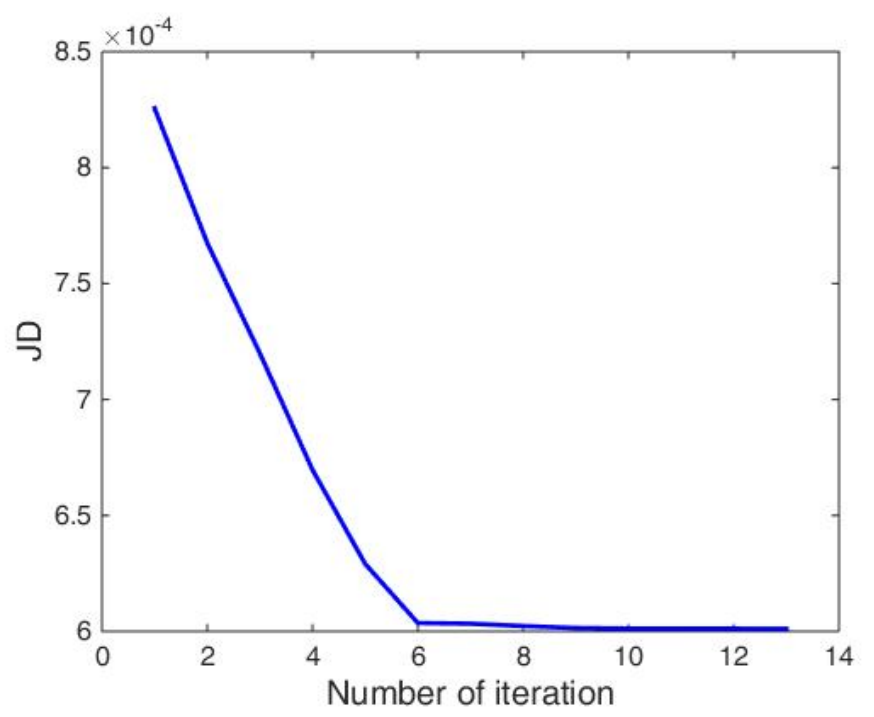

Figure 7. Gradient descent applied on the set of 2340 RMs computed using the II approach.

$\left.\begin{array}{|cc|cc}\hline \text { Stockman \& Sharp } & \multicolumn{3}{c|}{\text { Smith \& Pokorny }} \\ \left(\begin{array}{ccc}1.6934 & -1.5335 & 0.0750 \\ -0.8547 & 2.1269 & -0.2243 \\ 0.0215 & -0.0432 & 1.0169\end{array}\right)\end{array}\right)\left(\begin{array}{ccc}1.7191 & -1.5603 & 0.0666 \\ -0.8836 & 2.1573 & -0.1800 \\ 0.0349 & -0.0680 & 1.0128\end{array}\right)\left(\begin{array}{ccc}1.0388 & -0.3447 & 0 . \\ -0.1066 & 1.0896 & -0 \\ 0.0043 & -0.0137 & 1 .\end{array}\right.$

Table 2. Optimal global change of basis matrix found with the gradient descent for the three kinds of cone sensitivities.

where $0<\alpha_{n}<1$ is the step dependent weight of the numerical scheme.

The matrix which minimizes the error $J D$, among the 2340 matrices of our dataset with columns given by the eigenvectors of $\left\{A^{\mathbb{S}_{k}}\right\}$, was selected as the initial transformation $T_{0}$.

The stopping criterion of the numerical scheme is the following: we consider that the algorithm converged when the $L^{1}$ distance between $T_{n+1}$ and $T_{n}$ is less than $\varepsilon=10^{-3}$. This value for $\varepsilon$ was chosen empirically on the basis of the numerical values of the matrix elements of $T_{n}$ as well as for the efficiency of the algorithm.

In Fig. 7 we show the decreasing behavior of $J D\left(T_{n}\right)$ with respect to $n$.It can be seen that, after 6 iterations, the corrections are negligible. Thus, in practice, we can consider the algorithm to be convergent after 6 iterations with a value of JD of 0.00006 . This value is such that, on average, the off-diagonal elements of any matrix account for less than $0.4 \%$ of the sum of the absolute values of all of the matrix elements. In other words, this result empirically confirms that $T_{\text {opt }}$ almost diagonalize all matrices of our dataset.

Table 2 shows the $T_{o p t}$ found with the gradient descent for the three kinds of cone sensitivities. 


\subsection{Singularities in reflection properties and link with human color vi- sion}

In a natural environment, color is related to the reflectance properties of an object. Therefore, the geometrical properties of the RM should account for characteristics of human color vision.

\subsubsection{Singularities in reflection properties}

Contrary to the situation in physics where the space of the incident and reflected light has an infinite dimension (and therefore there are an infinity of reflection coefficients for each $\lambda$ ), in the biological case there are only three dimensions and, moreover, when placed in the virtual basis found by diagonalizing the RM, only three reflection coefficients (cf Eq. (7). This small number of reflection coefficients allow the presence of a few asymmetries and singularities.

One kind of singularity is observed in the case where all three coefficients are about equal, that is to say, when the surface transmits the incident light with a similar amplitude in all three non-interacting components of the virtual basis. This corresponds to the case of achromatic surfaces: black when the light is equally poorly transmitted $\left(r_{i}^{\mathbb{S}} \simeq 0\right)$, white when it is equally highly transmitted $\left(r_{i}^{\mathbb{S}} \simeq 1\right)$ and gray in the intermediate situation. Another type of singularity would happen when the surface's reflection only produce strong variations of the incoming light along one or two of the axis of the basis. In other words, a singularity would happen if one or two reflection coefficients $r_{i}^{\mathbb{S}}$ would be close to zero. In the first case, one reflection coefficient would be very large compared to the two others, whereas in the second case one reflection coefficient would be very small compared to the other two. Like achromatic surfaces, such singular surfaces are expected to bear a particular perceptual status.

In order to characterize the latter kind of singularities, PO defined a singularity index $\sigma^{\mathbb{S}}$ :

$$
\sigma^{\mathbb{S}}=\max \left(\frac{\sigma_{1}^{\mathbb{S}}}{\sigma_{1}^{\max }}, \frac{\sigma_{2}^{\mathbb{S}}}{\sigma_{2}^{\max }}\right)
$$

where $\sigma_{1}^{\mathbb{S}}$ is the ratio of the maximum reflection coefficient over the second maximum reflection coefficient for one surface, $\sigma_{2}^{\mathbb{S}}$ is the ratio of the second maximum reflection coefficient over the smallest reflection coefficient, and $\sigma_{1}^{\max }$ and $\sigma_{2}^{\max }$ are the maximum $\sigma_{1}^{\mathbb{S}}$ and $\sigma_{2}^{\mathbb{S}}$ values respectively over the entire dataset of surfaces. Maxima of $\sigma_{1}^{\mathbb{S}}$ correspond to sufaces that maximally reflect one of the incoming light's components in the virtual basis compared to the others two while maxima of $\sigma_{2}^{\mathbb{S}}$ correspond to surfaces that minimally reflects one of the incoming light's components in the virtual basis compared to the other two.

PO compared their computed singularity indexes with (Berlin and Kay 1991) psychophysical results using the same 320 colored munsell chips. They showed that the four chips with the highest local singularity index were only one chip far from the four chips which were most often given a name by widely separated human cultures (Fig 8) i.e. according to berlin1991basic, the four basic universal focal colors "red", "yellow", "green" and "blue". 
This result suggests that the largely cross-culturally spread particular perceptual status of these four surfaces, as being the prototypes of the four main color categories, and their occurrence in being named, may be a direct consequence of these surface reflection properties. All the more so since witzel2015determines showed how the singularity index is robust with respect to changes in cone sensitivity functions. Even though the latter observation requires further investigation to be fully understood, it remains a strong argument in favor of the universalists point of view regarding the color naming and linguistic relativity debate.

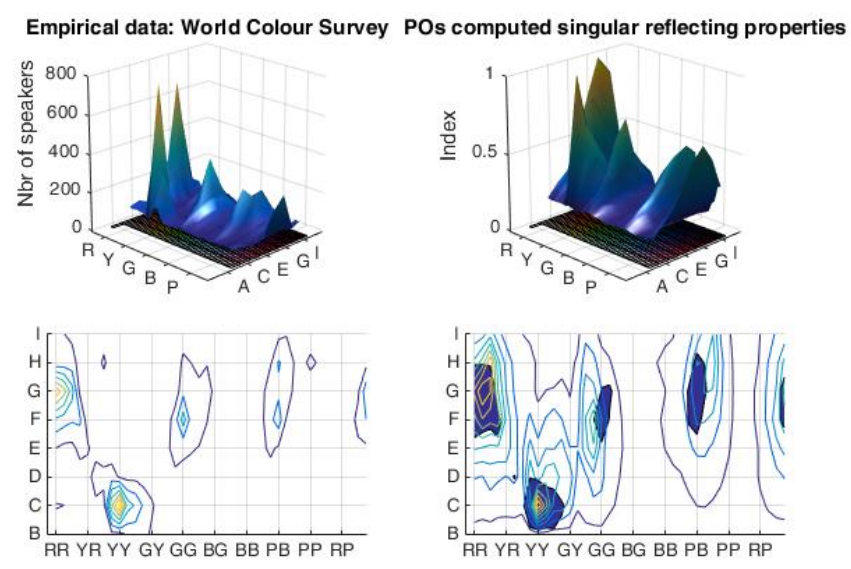

Figure 8. Singularity index computed with PO's approach on the set of 320 munsell chips of the WCS.

In addition, psychophysical studies on unique hues mostly rely on stimuli consisting in the direct presentation to the eyes of a highly spectrally controlled light, rather than natural viewing of a colored surface CITE. Even though their framework models surface reflectances as sensed by human eyes, PO were also able to relate the singularities of the RM with the four unique hues "red", "yellow", "green" and "blue". They did so by making the small conjecture that such stimuli are interpreted by the nervous system as being the result of a light reflected by a colored surface. Thus, it allowed them to transpose the index concerning surface properties into an index concerning photons caught by human photopigments (for the detailed transposition procedure, cf. (Philipona and O'Regan 2006)). Their transposed index enabled consistent predictions of psychophysical studies results on unique hues.

These results show that the properties of the computed RM may be a cause for characteristics of human color vision. Since we argued in section 0.3.3 that the RM computed with the II approach were more closely related to the action of a surface in the 3D space of human photoreceptors than with PO's, it is of interest to verify that the singularities of such RM still allow a good match with psychophysical studies. We only considered the link between singularity index and focal colors because focal colors and unique hues are so strongly related in PO's framework that observations on unique hues follow naturally from results on focal colors.

Figure $10 \mathrm{a}$ ) shows the singularity index obtained for the set of 320 surfaces of the WCS studies with the II approach performing a per surface diagonalization. We can see that the 

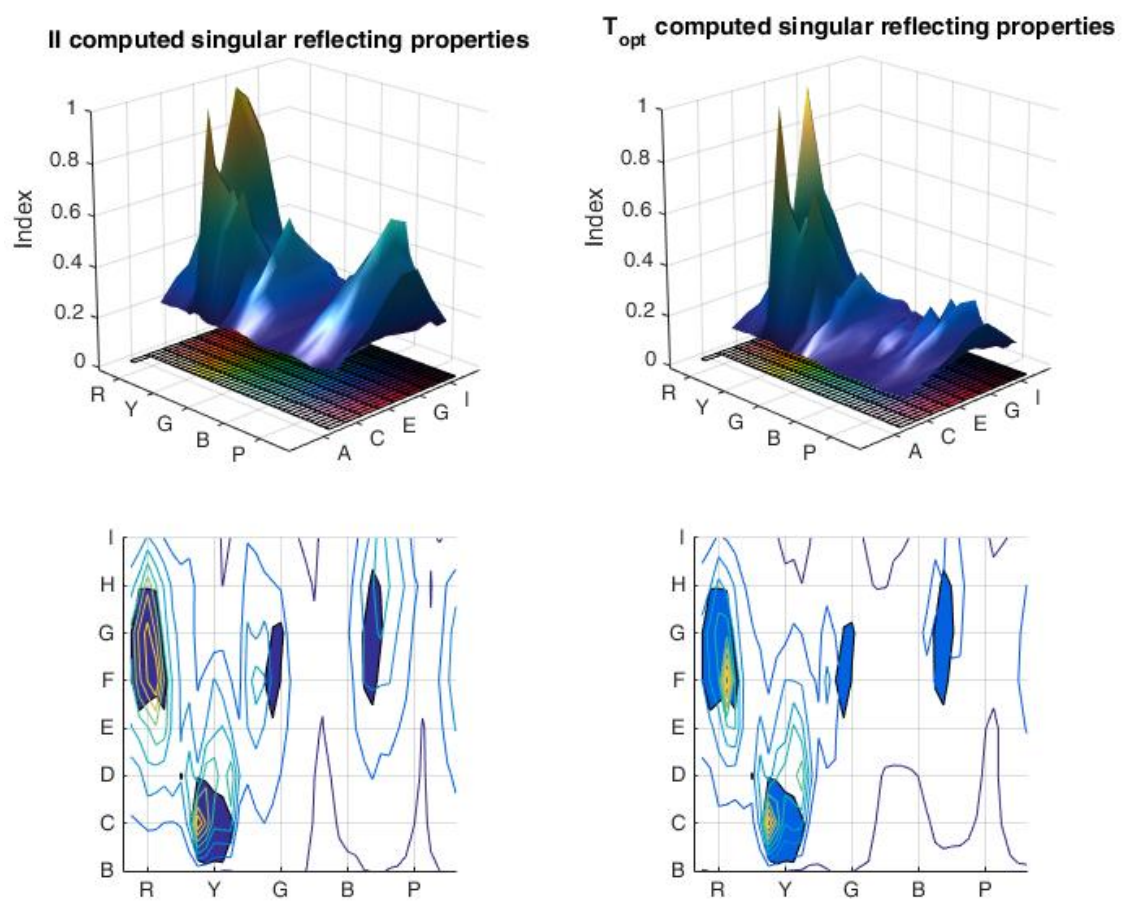

(a)

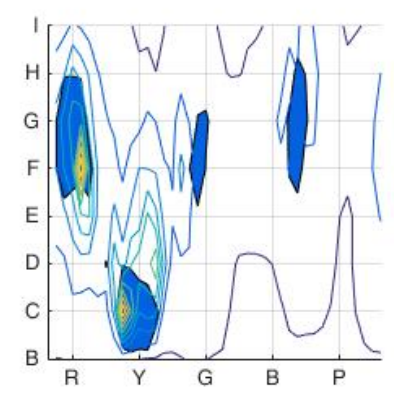

(b)

Figure 9. Singularity index of the 320 munsell chips computed with $a$ ) the II approach $b$ ) $T_{\text {opt }}$.

singularity index still correlates with the WCS results on color naming, with a correlation coefficient of 0.63 . The same can be observed for the index computed using a the single change of basis $T_{o p t}$, with this time however a correlation coefficient of 0.55 . In both cases, anyway, the predicted prototypes for the four main color categories remain very similar to the ones predicted by PO and observed in the WCS study.

\subsubsection{Analysis of biological reflectance singularities}

witzel2015determines extensively studied some characteristics of the singularity index, showing, among other things, that chroma and singularities are well correlated for the set of 320 Munsell chips, with a correlation coefficient of 0.7 .

Figure $10 \mathrm{a}$ ) displays the singularity index according to hues, values and chroma for our set of 1600 Munsell chips. Small and dark bluish dots corresponds to small values of the singularity index while large and lighter yellowish dots to high values of the singularity index. It appears clearly from this figure that chroma and singularities of munsell chips are very much correlated, while on the other hand value is not. Figure $10 \mathrm{~b}$ explicitly shows the dependence of the singularity of Munsell chips on chroma and value. They confirm the first impressions as well as Witzel et al.'s observations. The correlation between chroma 
and the singularity index is even higher in our case, with a correlation coefficient of 0.74 , while between value and the singularity index the correlation is of -0.1 . We find here also an explanation as to why, even for focal colors there is a disparity in singularity index values: some hues and values allow a higher range of saturation. YY and RR for instance allow chroma superior to 10-12, contrary to GG and BB.

This disparity in the influence of value and chroma on the singularity index can be qualitatively understood from figure 5. Recalling Eq. 12 we have as a first approximation: $S_{h, k v, c}(\lambda)=C(k) S_{h, v, c}(\lambda)$, where C is a multiplicative constant that depends on k. Thus in the framework of PO's model we can write:

$$
\begin{aligned}
\mathbf{v}^{\mathbb{S}_{h, k v, c}(E)} & =\int_{\Lambda} \mathbf{R}(\lambda) E(\lambda) S_{h, k v, c}(\lambda) d \lambda, \\
& =C(k) \int_{\Lambda} \mathbf{R}(\lambda) E(\lambda) S_{h, v, c}(\lambda) d \lambda, \\
& =C(k) A^{\mathbb{S}_{h, v, c} \mathbf{u}(E) .}
\end{aligned}
$$

which is equivalent to saying $A^{\mathbb{S}_{h, k v, c}}=C(k) A^{\mathbb{S}_{h, v, c}}$. Thanks to matrix properties, we have

$$
D^{\mathbb{S}_{h, k v, c}}=C(k) D^{\mathbb{S}_{h, v, c}}
$$

Since the singularity index only takes into account ratios between elements of the diagonal RM, the multiplicative constant $C(k)$ has no influence on the singularity index, which explains the very little influence of value itself.

On the other hand, we saw in section 0.3 .2 that chroma deforms the reflectance functions non-homogeneously, as seen in figure 5 b), by enhancing the contrast in reflectance between wavelengths. As a consequence, it will enlarge the contrast between reflection coefficients.

Another feature of singularities is their independence with respect to cone proportions on the retina. Cone proportions can be simply modeled by weights applied on cone sensitivities. Even though the weights can greatly modify the components of $\mathbf{u}(E)$ and $\mathbf{v}^{\mathbb{S}}(E)$ and change the components of the RM, they have no repercussion on their eigenvalues once in the virtual basis. This result is actually a consequence of the nature of the RM, as being a linear operator of the three dimensional space defined by the human cones. Indeed, applying a weight to cone sensitivities simply means applying an independent scaling to the components of the basis on which $\mathbf{u}(E)$ and $\mathbf{v}^{\mathbb{S}}(E)$ are expressed, without modifying the intrinsic properties of the linear operator. Interestingly, if we think of the relation between singularities of the RM and unique hues, this result is in accordance with the observations made by (Brainard et al. 2000) and (Pokorny and Smith 1987; Miyahara et al. 1998). They observed that the inter-individual differences in cone ratios, and especially for the $\mathrm{L}$ and $\mathrm{M}$ cones, had no influence on the perception of unique yellow. This observation further supports the idea that unique hues may be a consequence of reflection properties of surface reflectances, as they are sensed by human eyes.

In short, singularities in the biological reflectance properties seem closely linked to characteristics of human color perception, as they may account for the relationship between hue, 


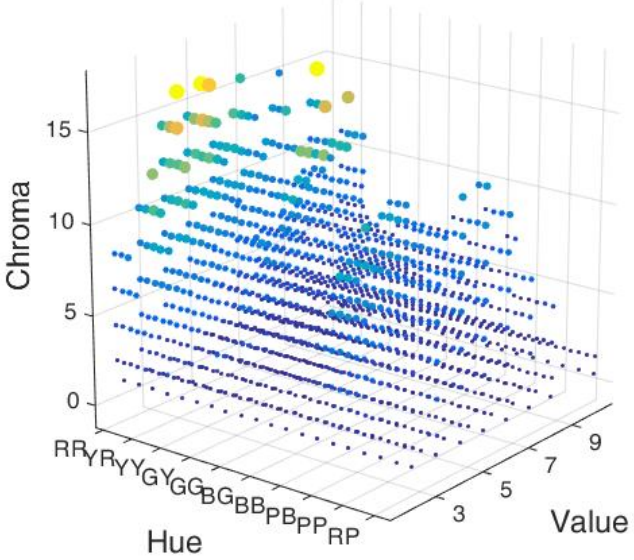

(a)
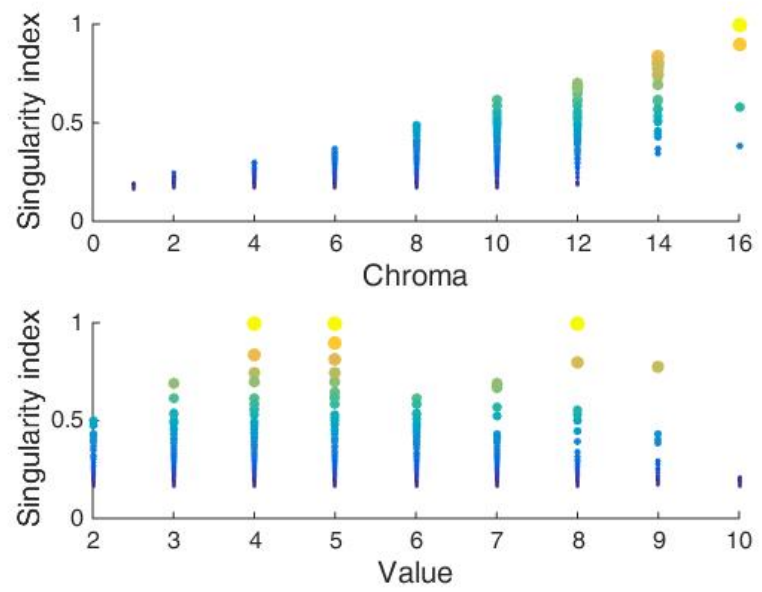

(b)

Figure 10. a) Singularity index according to hue, value and chroma for our set of 1600 Munsell chips. Small and dark bluish dots corresponds to small values of the singularity index while large and lighter yellowish dots to high values of the singularity index. $b$ ) Singularity index according to chroma and value. 
chroma and the particular perceptual status of colored surfaces. They also correlate with psychophysical studies on unique hues. On the other hand, singularities do not, or very poorly, account for the influence of the lightness of a surface in human color perception. In that aspect, singularities cannot fully account for the capacity of human color perception to categorize color.

\subsection{Conclusion}

We have shown in this work that an alternative method to PO's can be used to compute the $\mathrm{RM} A^{\mathbb{S}}$. This method has the advantage of being fully independent with respect to the illuminant. It is thus robust to illuminant change, while still allowing satisfactory results in the approximation of the information available to the eye about the reflected light $v^{\mathbb{S}}(E)$. It also allows the computation, through simple gradient descent, of a global change of basis transform $T$ which is independent of illuminants, and compatible with classic von Krieslike approaches to achieve color constancy despite the significant overlap of human cone sensitivity functions.

Another result we have obtained is to show that singularities of the RM computed with our approach still correlate with characteristics of human color vision, in particular its ability to categorize colors, and the independence of unique hues with respect to cone ratios. Furthermore, we showed that singularities accounted for hues and saturation, but were largely unaffected by variations in surface lightness. They thus cannot fully account for human's color categorization.

\subsection{Aknowledgment}

This work was financed by ERC Advanced grant "FEEL" Number 323674 to J. K. O’Regan. 


\section{Bibliography}

Afsari, B., and P. S Krishnaprasad. 2004. "Some gradient based joint diagonalization methods for ICA". In Independent Component Analysis and Blind Signal Separation, 437-444. Springer.

Berlin, B., and P. Kay. 1991. Basic color terms: Their universality and evolution. Univ of California Press.

Brainard, D. H, J. M Kraft, and P. Longere. 2003. "Color constancy: Developing empirical tests of computational models". Colour perception: From light to object: 307-334.

Brainard, D. H, A. Roorda, Y.i Yamauchi, J. B Calderone, A. Metha, M. Neitz, J. Neitz, D. R Williams, and G. H Jacobs. 2000. "Functional consequences of the relative numbers of $\mathrm{L}$ and M cones". JOSA A 17 (3): 607-614.

Brill, M., and G. West. 1981. "Contributions to the theory of invariance of color under the condition of varying illumination". Journal of Mathematical Biology 11 (3): 337-350.

Bunse-Gerstner, A., R. Byers, and V. Mehrmann. 1993. "Numerical methods for simultaneous diagonalization”. SIAM Journal on Matrix Analysis and Applications 14 (4): 927-949.

Cardoso, J-F., and A. Souloumiac. 1996. "Jacobi angles for simultaneous diagonalization". SIAM journal on matrix analysis and applications 17 (1): 161-164.

Chiao, C-C., T. W Cronin, and D. Osorio. 2000. "Color signals in natural scenes: characteristics of reflectance spectra and effects of natural illuminants". JOSA A 17 (2): 218-224.

Finlayson, G. D, M. S Drew, and B. V Funt. 1994. "Spectral sharpening: sensor transformations for improved color constancy”. JOSA A 11 (5): 1553-1563.

Forsyth, D. A. 1990. “A novel algorithm for color constancy". International Journal of Computer Vision 5 (1): 5-35.

Foster, David H. 1984. “Colour vision”. Contemporary Physics 25 (5): 477-497.

Gelfand, I. M, R. A Silverman, et al. 2000. Calculus of variations. Courier Corporation.

Gijsenij, A., T. Gevers, and J. Van De Weijer. 2011. "Computational color constancy: Survey and experiments". Image Processing, IEEE Transactions on 20 (9): 2475-2489.

Hori, G. 1999. "Joint diagonalization and matrix differential equations". Proc. of NOLTA'99: 675-678.

Jiang, J., D. Liu, J. Gu, and S. Susstrunk. 2013. "What is the space of spectral sensitivity functions for digital color cameras?" In Applications of Computer Vision (WACV), 2013 IEEE Workshop on, 168-179. IEEE.

Jraissati, Y. 2014. "Proving universalism wrong does not prove relativism right: Considerations on the ongoing color categorization debate". Philosophical psychology 27 (3): 401424. 
Judd, D. B, D. L MacAdam, G. Wyszecki, HW Budde, HR Condit, ST Henderson, and JL Simonds. 1964. "Spectral distribution of typical daylight as a function of correlated color temperature". Josa 54 (8): 1031-1040.

Koenderink, JJ, and AJ van Doorn. 2003. Perspectives on colour space.

Land, E. H. 1964. "The retinex”. American Scientist 52 (2): 247-264.

1983. "Recent advances in retinex theory and some implications for cortical computations: color vision and the natural image". Proceedings of the National Academy of Sciences 80 (16): 5163-5169.

Land, E. H, and J. J McCann. 1971. "Lightness and retinex theory". JOSA 61 (1): 1-11.

Logvinenko, AD, B. Funt, H. Mirzaei, and R. Tokunaga. 2015. "Rethinking colour constancy". PloS one 10 (9): e0135029.

Malacara, Daniel. 2011. "Color vision and colorimetry: theory and applications". SPIE.

Miyahara, E., J. Pokorny, Vivianne C. S., Baron R., and E/ Baron. 1998. "Color vision in two observers with highly biased LWS/MWS cone ratios". Vision Research 38 (4): 601-612.

O'Meara, KC, and C Vinsonhaler. 2006. "On approximately simultaneously diagonalizable matrices”. Linear algebra and its applications 412 (1): 39-74.

Parkkinen, J. PS, J Hallikainen, and T Jaaskelainen. 1989. “Characteristic spectra of Munsell colors". JOSA A 6 (2): 318-322.

Philipona, D. L, and J K. O'Regan. 2006. "Color naming, unique hues, and hue cancellation predicted from singularities in reflection properties". Visual neuroscience 23 (3-4): 331-339.

Pokorny, J, and VC Smith. 1987. "L/M cone ratios and the null point of the perceptual red/green opponent system". Die Farbe 34:53-57.

Provenzi, E., C. Gatta, M. Fierro, and A. Rizzi. 2008. "A spatially variant white-patch and gray-world method for color image enhancement driven by local contrast". Pattern Analysis and Machine Intelligence, IEEE Transactions on 30 (10): 1757-1770.

Romero, J., A. Garcia-Beltrán, and J. Hernández-Andrés. 1997. "Linear bases for representation of natural and artificial illuminants". JOSA A 14 (5): 1007-1014.

Smith, V. C, and J. Pokorny. 1975. "Spectral sensitivity of the foveal cone photopigments between 400 and $500 \mathrm{~nm}$ ". Vision research 15 (2): 161-171.

Stiles, W. S., and J. M Burch. 1959. "NPL colour-matching investigation: final report (1958)". Journal of Modern Optics 6 (1): 1-26.

Stockman, A., and L. T Sharpe. 2000. "The spectral sensitivities of the middle-and longwavelength-sensitive cones derived from measurements in observers of known genotype". Vision research 40 (13): 1711-1737.

Tichavsk, P., and A. Yeredor. 2009. "Fast approximate joint diagonalization incorporating weight matrices”. Signal Processing, IEEE Transactions on 57 (3): 878-891. 
Van De Weijer, J., and Th Gevers. 2005. "Color constancy based on the grey-edge hypothesis”. In Image Processing, 2005. ICIP 2005. IEEE International Conference on, 2:II-722. IEEE.

Vazquez-Corral, J., J K. O’Regan, M. Vanrell, and G. D Finlayson. 2012. “A new spectrally sharpened sensor basis to predict color naming, unique hues, and hue cancellation". Journal of vision 12 (6): 7-7.

Von Kries, J. 1902. "Chromatic adaptation”. Festschrift der Albrecht-Ludwigs-Universität 135:145-158.

Wandel, BA. 1995. "Foundation of vision sinauer associates". Inc. Sunderland MA.

West, G., and M. H Brill. 1982. "Necessary and sufficient conditions for von Kries chromatic adaptation to give color constancy". Journal of Mathematical Biology 15 (2): 249-258.

Westland, S., J. Shaw, and H. Owens. 2000. "Colour statistics of natural and man-made surfaces". Sensor Review 20 (1): 50-55.

Witzel, C., F. Cinotti, and J K. O'Regan. 2015. "What determines the relationship between color naming, unique hues, and sensory singularities: Illuminations, surfaces, or photoreceptors?" Journal of vision 15 (8): 19-19.

Wyszecki, G Stiles, and W Stiles. 1982. WS,(1982), Color Science: Concepts and Methods, Quantitative Data and Formulae.

Ziehe, A., P. Laskov, G. Nolte, and K-R. Müller. 2004. "A fast algorithm for joint diagonalization with non-orthogonal transformations and its application to blind source separation". The Journal of Machine Learning Research 5:777-800.

\subsection{Appendicies}

\subsubsection{Appendix A: Linear and Illuminant-Independant hypothesis}

Let $\bar{\Lambda}=\left[\lambda_{1}, \lambda_{2}\right], \Lambda \subset \bar{\Lambda}$, such that $E\left(\lambda_{1}\right)=E\left(\lambda_{2}\right)=0, \forall E(\lambda)$. Every $E(\lambda)$ is considered smooth (continuous is sufficient here). Then According to the fundamental Lemma of Calculus of Variation :

$$
\int_{\bar{\Lambda}} d \lambda E(\lambda)\left[S(\lambda) R_{i}(\lambda)-\sum_{j} A_{i j}^{\mathbb{S}} R_{j}(\lambda)\right]=0, \quad \forall \lambda \in \bar{\Lambda}
$$

is equivalent to :

$$
S(\lambda) R_{i}(\lambda)-\sum_{j} A_{i j}^{\mathbb{S}} R_{j}(\lambda)=0, \quad \forall \lambda \in \bar{\Lambda}
$$

Now, as $R_{i}(\lambda)=0, \forall \lambda \notin \Lambda, \quad i=L, M, S$, then equation 30 is equivalent to :

$$
S(\lambda) R_{i}(\lambda)-\sum_{j} A_{i j}^{\mathbb{S}} R_{j}(\lambda)=0, \quad \forall \lambda \in \Lambda
$$


Thus a linear operator $A^{\mathbb{S}}$ such that $v^{\mathbb{S}}(E)=A^{\mathbb{S}} u(E)$, independently on $E(\lambda)$ exists if and only if there exist $\left\{A_{i j}^{\mathbb{S}}\right\}$ such that :

$$
S(\lambda) R_{i}(\lambda)=\sum_{j} A_{i j}^{\mathbb{S}} R_{j}(\lambda), \quad i, j=L, M, S, \quad \forall \lambda \in \Lambda
$$

where $\left\{A_{i j}\right\}$ are the components of $A^{\mathbb{S}}$.

\subsubsection{Appendix B: Relative norm of a commutator}

Let $A_{1}$ and $A_{2}$ denote two matrices. Their commutator is defined as $C_{12}=A_{1} A_{2}-A_{2} A_{1}$. Two properties of matrix norms are the following:

$$
\left\|A_{1}+A_{2}\right\| \leq\left\|A_{1}\right\|+\left\|A_{2}\right\|
$$

and

$$
\left\|A_{1} A_{2}\right\| \leq\left\|A_{1}\right\|\left\|A_{2}\right\|
$$

We then have :

$$
\begin{aligned}
\left\|C_{12}\right\| & =\left\|A_{1} A_{2}-A_{2} A_{1}\right\| \\
& \leq\left\|A_{1} A_{2}\right\|+\left\|A_{2} A_{1}\right\| \\
& \leq\left\|A_{1}\right\|\left\|A_{2}\right\|+\left\|A_{2}\right\|\left\|A_{1}\right\| \\
& \leq 2\left\|A_{1}\right\|\left\|A_{2}\right\|
\end{aligned}
$$

Which is in turn equivalent to :

$$
\frac{\left\|C_{12}\right\|}{2\left\|A_{1}|| A_{2}\right\|} \leq 1
$$

